\title{
Comorbid Problems in ADHD: Degree of Association, Shared Endophenotypes, and Formation of Distinct Subtypes. Implications for a Future DSM
}

\author{
Nanda N. J. Rommelse • Marieke E. Altink • Ellen A. Fliers • Neilson C. Martin • \\ Cathelijne J. M. Buschgens • Catharina A. Hartman • Jan K. Buitelaar • \\ Stephen V. Faraone · Joseph A. Sergeant • Jaap Oosterlaan
}

Published online: 24 March 2009

(C) The Author(s) 2009. This article is published with open access at Springerlink.com

\begin{abstract}
We aimed to assess which comorbid problems (oppositional defiant behaviors, anxiety, autistic traits, motor coordination problems, and reading problems) were most associated with Attention-Deficit/Hyperactivity Disorder (ADHD); to determine whether these comorbid problems shared executive and motor problems on an endophenotype level with ADHD; and to determine whether executive
\end{abstract}

Electronic supplementary material The online version of this article (doi:10.1007/s10802-009-9312-6) contains supplementary material, which is available to authorized users.

N. N. J. Rommelse · J. A. Sergeant · J. Oosterlaan

Department of Clinical Neuropsychology,

VU University Amsterdam,

Amsterdam, The Netherlands

N. N. J. Rommelse $(\bowtie) \cdot$ M. E. Altink $\cdot$ E. A. Fliers

C. J. M. Buschgens $\cdot$ J. K. Buitelaar

Department of Psychiatry, Radboud University Medical Centre,

Reinier Postlaan 12, 6525 GC,

Nijmegen, The Netherlands

e-mail: n.lambregts-rommelse@psy.umcn.nl

E. A. Fliers

Youth Department, BAVO-Europoort,

Rotterdam, The Netherlands

N. C. Martin

School of Psychology, Curtin University of Technology,

Perth, Western Australia

C. A. Hartman

Department of Psychiatry, University of Groningen,

Groningen, The Netherlands

S. V. Faraone

Departments of Psychiatry and Neuroscience \& Physiology,

SUNY Upstate Medical University,

Syracuse, NY, USA functioning (EF) - and motor-endophenotypes supported the hypothesis that ADHD with comorbid problems is a qualitatively different phenotype than ADHD without comorbid problems. An EF-and a motor-endophenotype were formed based on nine neuropsychological tasks administered to 816 children from ADHD - and control-families. Additional data on comorbid problems were gathered using questionnaires. Results indicated that oppositional defiant behaviors appeared the most important comorbid problems of ADHD, followed by autistic traits, and than followed by motor coordination problems, anxiety, and reading problems. Both the EF- and motor-endophenotype were correlated and cross-correlated in siblings to autistic traits, motor coordination problems and reading problems, suggesting ADHD and these comorbid problems may possibly share familial/genetic EF and motor deficits. No such results were found for oppositional defiant behaviors and anxiety. ADHD in cooccurrence with comorbid problems may not be best seen as a distinct subtype of ADHD, but further research is warranted.

Keywords Attention-deficit/Hyperactivity disorder. Comorbidity $\cdot$ Endophenotype $\cdot$ Phenotype $\cdot D S M-V$

Attention-Deficit/Hyperactivity Disorder (ADHD) (American Psychiatric Association [APA] 1994) is frequently associated with a range of other psychiatric and neurological disorders. It is estimated that around $60-100 \%$ of patients with ADHD also exhibit one or more comorbid disorders (Gillberg et al. 2004) that often continue into adulthood (Biederman 2004; Kessler et al. 2006). Around $42-90 \%$ of patients meet criteria for Oppositional Defiant Disorder (ODD) and/or Conduct Disorder (CD) (Angold et al. 1999; Bauermeister et al. 2007; Cunningham and Boyle 2002; Gillberg et al. 
2004; Jensen et al. 1997), disorders characterized by externalizing behavioral problems, such as aggressive behavior, difficulty with authority (ODD) or lying, stealing, and vandalism (CD). Furthermore, around $13-51 \%$ of ADHD patients suffer from internalizing disorders, such as anxiety or depression (Angold et al. 1999; Bauermeister et al. 2007; Gillberg et al. 2004; Jensen et al. 1997). Currently, the Diagnostic and Statistical Manual of Mental Disorders (DSM) IV (APA 1994) rules out a diagnosis of autistic disorder with ADHD. Nevertheless, a large percentage $(65-80 \%)$ of children with ADHD portrays symptoms in the autistic spectrum (Clark et al. 1999; Gillberg et al. 2004). Other disorders frequently observed in patients with ADHD, are dyslexia (25-40\%), motor coordination problems $(50 \%)$, dyscalculia (10-60\%), sleep disorders (25-50\%), and enuresis and/or encopresis (30\%) (Bhatia et al. 1991; Gillberg et al. 2004; Owens 2005; Willcutt et al. 2005). ADHD patients with comorbid problems compared to ADHD patients without comorbid problems appear to have a more severe form of ADHD, are often more impaired in their daily functioning, and have a poorer long term prognosis (Bauermeister et al. 2007; Biederman et al. 1996; Connor et al. 2003; Gillberg et al. 2004). This may have implications for diagnosis and treatment of ADHD, like broad assessments covering multiple childhood psychiatric disorders and not only ADHD and interventions that also address the comorbid problems (Biederman et al 1991; Gillberg et al. 2004; Hechtman et al. 2005; Jensen et al. 2001).

Clearly, comorbid problems are an important aspect of ADHD. However, the word 'comorbid' has various meanings. For example, it may refer to one disorder leading to another, it may refer to two (or more) clearly separable and independent disorders occurring together, or it can refer to two (or more) disorders that share a common underlying etiology (Angold et al. 1999; Biederman et al. 1991; Caron and Rutter 1991; Gillberg et al 2004). Understanding how comorbidity arises may inform our understanding of the development of psychopathology (Angold et al. 1999). In light of the development of a future DSM, studying the degree and nature of the association between ADHD and its comorbid problems deserves further attention (Jensen et al. 1997). The current study was designed to examine whether ADHD and several comorbid disorders share a common underlying etiology.

Therefore, the first aim of our study was to investigate the degree of association between ADHD and several comorbid problems (oppositional defiant behaviors, anxiety, autistic traits, motor coordination problems, and reading problems). We analyzed which comorbid problem was the most strongly related to ADHD. To assess this, we used questionnaires. Questionnaires generally correlate strongly with structured interviews (Biederman et al. 2005; Conners 1996) and may aptly reflect the underlying continuous distribution of traits (Reich et al. 1975). Using questionnaires, it was possible to estimate the degree of phenotypic association between ADHD and its comorbid problems.

The second aim of our study was to clarify whether ADHD and comorbid problems are 'merely' associated with each other at a phenotypic level or whether they partly arise from the same heritable, vulnerability traits. In previous studies (Rommelse et al. 2007a, b, c, 2008a, b), we have identified neuropsychological endophenotypes for ADHD: heritable deficits that formed underlying, vulnerability traits for ADHD. Characteristic of endophenotypes is that they are also present in at risk non-affected familymembers of ADHD patients and show resemblance between family-members (Waldman 2005). These endophenotypes may shed light on the nature of the association between ADHD and its comorbid problems: if the endophenotype also relates to the comorbid problems (while correcting for ADHD), it is likely that both disorders (partly) relate to the same underlying neuropsychological substrate and are not merely phenotypically associated with one another. Further evidence for this hypothesis may be obtained, when these neuropsychological endophenotypes cross-correlate with comorbid problems in siblings (i.e. neuropsychological deficits of a child relate to comorbid problems in his/her siblings), suggesting similar familial (and possibly genetic) influences give rise to ADHD and comorbid problems. Thus, these correlations and sibling crosscorrelations may indicate whether or not ADHD and comorbid problems partly arise from the same familial/genetic neuropsychological vulnerabilities. The neuropsychological endophenotypes used in this study comprise both executive functioning (EF) and motor functioning, two areas of neuropsychological impairment most strongly related to ADHD.

The third aim of our study was to investigate whether the specific combination of ADHD with a comorbid problem may be seen as forming a distinct phenotype (Banaschewski et al. 2005; Biederman et al. 1991; Caron and Rutter 1991; Jensen et al. 2001) and not merely as 'more of the same of both disorders'. If the comorbid condition is a third, independent condition (Caron and Rutter 1991), it is expected that the interaction term between ADHD and the comorbid condition will have predictive value on the neuropsychological traits beyond the independent effects of ADHD and the comorbid condition (Baron and Kenny 1986).

In sum, we aimed to assess (1) which comorbid problems (oppositional defiant behaviors, anxiety, autistic traits, motor coordination problems, and reading problems) were most associated with ADHD, (2) whether these comorbid problems share familial (possibly genetic) EF and motor vulnerabilities (endophenotypes) with ADHD, and (3) whether EF-and motor-endophenotypes supported the hypothesis that ADHD with comorbid problems is a qualitatively different phenotype than ADHD without 
comorbid problems. Even though unraveling the nature of the comorbidity between ADHD and other problems is far from new, the current study adds to the existing findings by taking a new approach. That is, to the best of our knowledge, this is the first study that uses a neuropsychological endophenotypical approach to examine whether ADHD and its comorbidities arise from similar, heritable, neuropsychological dysfunctions underlying behavioral symptoms.

\section{Materials and Methods}

\section{Participants}

Participants were recruited in the Dutch part of an international multicenter ADHD genetic study (IMAGE) that aims to identify genes that increase the risk for ADHD using quantitative trait loci linkage and association strategies (Brookes et al. 2006). Families with at least one child with the combined subtype of ADHD (proband) and at least one additional sibling (regardless of possible ADHD-status) participated. Probands were clinically referred to specialist centres. Additional control families were recruited from primary and high schools from the same geographical regions as the participating ADHD-families. Controls and their first degree relatives were required to have no formal or suspected ADHD diagnosis. A total of 238 ADHD-families and 147 control-families fulfilled inclusion and exclusion criteria. A total of 12 families from the original 250 ADHDfamilies were excluded based on the inclusion and exclusion criteria after positive screening for ADHD in the proband. Within the ADHD-families, 238 probands (all with combined subtype ADHD), 112 affected siblings (64 with combined subtype, 28 with inattentive subtype and 20 with hyperactive-impulsive subtype) and 195 non-affected siblings participated. Control-families consisted of 271 children. For 51 control children, no additional control sibling could be recruited for the study. The groups did not differ in age, but the group of probands and affected siblings had a larger percentage of males than the groups of non-affected siblings and controls (probands: $M$ age $=12.0(2.5), \%$ males $=84.5$; affected siblings: $M$ age=12.0 (3.4), \% males=56.3; nonaffected siblings: $M$ age=11.5 (3.6), \% males $=45.1$; controls: $M$ age $=11.6(3.2), \%$ males=40.6). All children were between the ages of 5 and 19 years and were of European Caucasian descent. Participants were excluded, if they had an IQ $<70$, a diagnosis of autism, epilepsy, acquired traumatic brain injury or known genetic disorders, such as Down syndrome or Fragile-X-syndrome.

The exact screening procedures and measures for ADHD phenotyping have been described previously (Brookes et al. 2006). Briefly, screening questionnaires (parent and teacher Conners' long version rating scales [Conners 1996] and parent and teacher Strengths and Difficulties Questionnaires (SDQ) [Goodman 1997]) were used to identify children with ADHD symptoms. Scores were considered clinical if $T$-scores were obtained $\geq 63$ on at least one Conners' ADHD-subscale (DSM-IV Inattention, DSM-IV Hyperactive-Impulsive, and $D S M-I V$ ADHD Total) or scores $>90$ th percentile on the SDQ-hyperactivity scale. Additionally, a semistructured interview, the Parental Account of Children's Symptoms (PACS) (Taylor 1986), was administered for children scoring clinically on any of the questionnaire scales of interest. For diagnostic purposes, questionnaire data and the PACS were subjected to a standardised algorithm to derive each of the 18 DSM-IV ADHD symptoms, providing operational definitions for each behavioral symptom (see for a description Rommelse et al. 2007b). The section on autistic traits of the PACS was administered to exclude possible cases with autistic disorder, if a clinical score (raw score $\geq 15$ ) was obtained on the Social Communication Questionnaire (SCQ; Berument et al. 1999). Concerning control children, the Conners' long version for both parents and teachers was completed and control children were required to obtain nonclinical scores on all scales measuring ADHD related symptomatology. A measure of ADHD for further analyses was operationalized by averaging the scaled Conners' $\mathrm{N}$ subscale (ADHD Total) for the parent and teacher rating.

This sample of children allowed for analyses on continuously distributed data on ADHD and comorbid problems, since they represented the whole range of possible scores on the Conners' ADHD scales ( $T$-scores from 40 to 90: control children had $T$-scores less than 62 , probands had $T$-scores of 63 and over, and siblings of probands had scores in the whole range) and since no disorders were excluded in the screening, except a full diagnosis of autism.

\section{Measures}

\section{Data on Comorbid Problems}

Oppositional Defiant Behaviors The Conners' Long Version filled out by the parents and teachers was used to get an indication of oppositional defiant behaviors (Subscale A) (Conners 1996). Subscale A consisted of ten items that were rated on a 4 -point scale $(0=$ not true at all; $3=$ very much true). Higher scores were indicative of more oppositional defiant behaviors. Reliability and validity of the Conners' have been established (Conners 1996). A measure of oppositional defiant behaviors was operationalized by averaging the scaled Conners' A-subscale (Oppositional) for the parent and teacher rating.

Anxiety The Conners' Long Version was used to obtain a measure of anxiety (Subscale D), both as observed by parents and teachers (Conners 1996). Subscale D consisted 
of eight items that were rated on a 4 -point scale $(0=$ not true at all; $3=$ very much true). Higher scores were indicative of more symptoms of anxiety. A measure of anxiety was operationalized by averaging the scaled Conners' Dsubscale (Anxious-Shy) for the parent and teacher rating.

Autistic Traits Ratings on symptoms in the autism spectrum were obtained using the Dutch 49-item Children's Social Behavior Questionnaire (CSBQ) (Hartman et al. 2006). Parents were asked to rate the behaviors of their child on a 3 -point scale $(0=$ not; $2=$ clearly/often $)$. The questionnaire consists of several subscales: Unadjusted behavior, Tendency to withdrawal, Orientation problems, Difficulty understanding, Stereotype movements, and Anxiety for changes. The reliability and validity of this questionnaire have been established (Hartman et al. 2006). The total sum score was used as a measure of symptoms in the autism spectrum, with higher scores indicative of more severe autistic like behaviors. Importantly, children with a full autistic disorder were excluded from the study, since these children cannot be diagnosed with ADHD according to the DSM-IV. Our findings on autistic traits are thus limited to children with sub clinical autistic disorder.

Motor Coordination Problems Motor coordination problems were rated by parents and teachers. Parents filled out the Developmental Coordination Disorder Questionnaire (DCD-Q) (Wilson et al. 2000). The DCD-Q consists of 17 items that were rated on a 5 -point scale $(1=$ not at all like this child; $5=$ extremely like this child). The total score was used as an indication of motor coordination problems, with a low score reflective of more severe motor coordination problems. The reliability and validity of this questionnaire have been confirmed (Wilson et al. 2000). Teachers rated motor coordination problems on the 17-item Groninger Motor Observation-scale (GMO) (Kalverboer and Van Dellen 1990), using a 4-point scale (1=not applicable; 4= applicable). Items related both to fine and gross motor coordination. The total score was used as indication of motor coordination problems, with higher scores reflecting more severe problems. The reliability and validity of this questionnaire have been established (Kalverboer and Van Dellen 1990). A measure of motor coordination problems was operationalized by averaging the standardized total scores for the parent and teacher rating.

Reading Problems Reading problems were assessed with the 6-item questionnaire of Willcutt and colleagues filled out by the parents (Willcutt et al. 2008). A 5-point scale was used $(1=$ never/not at all; $5=$ always/a great deal). A higher score reflected more severe reading problems. The total score of this questionnaire is known to correlate between 0.61 and 0.71 with validated measures of reading problems (Willcutt et al. 2009) and was used as measure of reading problems.

\section{Neuropsychological Tasks}

The nine experimental tasks described in this study have been fully described elsewhere (Rommelse et al. 2007a, b, c, 2008a, b). A short description of each task is given below. Based on previous results (Rommelse et al. 2007a, b, c, $2008 \mathrm{a}, \mathrm{b})$, the task variable that showed the most optimal result in the endophenotypic analyses in the five previous studies was chosen for the current analyses. These task variables were significantly associated with ADHD (i.e. affected children performed more poorly than control children) and were significantly correlated between siblings.

\section{Executive Tasks}

Stop Task The Stop Task was used to measure speed of inhibition of an ongoing response (Rommelse et al. 2008b). Subjects were presented two types of trials: go-trials and stop-trials. Go-trials consisted of the presentation of a gostimulus (drawing of a plane) that was either pointing to the right or to the left. Children were instructed to press a response button that corresponded to the direction of the stimulus as quickly and as accurately as possible. Stop-trials were identical to the go-trials but in addition a stop-signal was presented (drawing of a cross that was superimposed on the plane). Children were required to withhold their response to the stop-signal. Go stimuli were displayed for $1,000 \mathrm{~ms}$, preceded by a $500 \mathrm{~ms}$ fixation point. Stop signals were displayed for 1,000 ms minus delay time. Inter-trial intervals were 3,000 ms. The delay between the go-and stop-signal was dynamically varied so that it could be estimated when the child successfully inhibited $50 \%$ of the stop-trials, and unsuccessfully inhibited the other $50 \%$. At this point, the goprocess and stop-process were of equal duration, which made it possible to estimate the latency of the stop-process: the Stop Signal Reaction Time (SSRT). A total of 2 practice blocks and 4 experimental blocks were administered, each consisting of 60 trials. The first practice block consisted of only go-trials. The second practice block and the 4 experimental blocks consisted of $75 \%$ go-trials and $25 \%$ stop-trials. Go - and stop-trials were pseudo-randomly presented. Task administration took about $15 \mathrm{~min}$. Based on previous results (Rommelse et al. 2008b), the dependent measure was the SSRT, which showed endophenotypic-like group differences and correlated between siblings.

Shifting Attentional Set Shifting Attentional Set was designed to measure the accuracy of motor inhibition and cognitive flexibility (Rommelse et al. 2007a). The task consisted of three blocks of which the first block was 
designed to acquire a baseline of the accuracy of responding with which the performance on the second (motor inhibition) and third (cognitive flexibility) block could be compared. In all blocks, trials consisted of a horizontal bar with ten grey squares presented permanently at the centre of the screen. From trial to trial, a coloured square moved across the bar in a random direction (either one square to the right or to the left). Responses were required to be initiated between 150 to $5,000 \mathrm{~ms}$ after a square moved one position, otherwise a trial was replaced. The task was self-paced with post-response intervals of $250 \mathrm{~ms}$. In the first block, the moving square was coloured green, and compatible responses were required: Children were instructed to press a response button as quickly and as accurately as possible that corresponded to the direction in which the stimulus moved. In the second block, the moving square was coloured red, and incompatible responses were required. The suppression of the automatic compatible response, in order to generate a non-automatic incompatible response, was hypothesized as requiring inhibitory control. In the third block, the colour of the moving square alternated randomly between green and red, and both compatible and incompatible responses were required. Thus, both the direction and the colour of the square were unpredictable. This mixture of both compatible and incompatible trials was hypothesized as requiring high levels of cognitive flexibility in addition to inhibitory control. The first and second block consisted of 10 practice trials and 40 experimental trials. The third block consisted of 16 practice trials and 80 experimental trials. Administration took about 10 to $15 \mathrm{~min}$. The dependent measure was the percentage of errors across blocks measuring overall inaccuracy on cognitive taxing tasks, which was the best indicator of endophenotypic vulnerabilities (Rommelse et al. 2007a).

Visuo-Spatial Sequencing The Visuo-Spatial Sequencing task was used to measure accuracy of visuo-spatial working memory (Rommelse et al. 2008b). Stimuli consisted of nine circles symmetrically organized in a square ( 3 by 3 ). On each trial, a sequence of circles was pointed at by a computerdriven hand. Subjects were instructed to replicate the exact same sequence of circles, by pointing to them with the small, self-driven hand. There were no time constrictions. One practice trial and 24 experimental trials were presented. Every succeeding trial increased in difficulty level: an increase in the number of circles required to be remembered and/or an increase in the complexity of the spatial pattern (i.e. the trial consisted of circles that were spatially further removed from one another instead of being close to one another), hence manipulating working memory demands. Task administration took about $7 \mathrm{~min}$. Based on previous results, the total number of correct targets in the correct order was used as dependent measure reflecting endophenotypic-like group differences and correlating between siblings (Rommelse et al. 2008b).
Digit Span The Digit Span backwards of the WISC-III and WAIS-III was used to obtain an indication of verbal working memory (Rommelse et al. 2008b). The task consisted of repeating a sequence of numbers in the opposite order. Children were instructed to reproduce sequences as accurately as possible. One digit was added to the sequence if a child reproduced the sequence successfully. Two practice trials with a 2 digit sequence and (dependent on the child's performance) a maximum of 8 experimental sequences were administered. Dependent measure was the maximum Digit Span backwards, which proved useful as endophenotypic candidate (Rommelse et al. 2008b).

\section{Motor Tasks}

Pursuit This task was designed to measure precision of motor control under continuous adaptation (Rommelse et al. 2007c). The stimulus consisted of a randomly moving target (asterisk) that was required to be 'caught' by moving a mouse cursor on top of the asterisk. The target moved at a constant speed of $10 \mathrm{~mm} / \mathrm{s}$. Children were instructed to 'catch' the randomly moving target as precisely as possible. One practice (13 s) and one experimental session (60 s) were administered for both hands separately. Administration took about $5 \mathrm{~min}$. The dependent measure was the precision (mean distance in $\mathrm{mm}$ between target and cursor calculated per second and averaged across the $60 \mathrm{~s}$ experimental session) of the left hand. Previous results have shown that mainly the performance of the left hand was most strongly associated with ADHD (Rommelse et al. 2007c).

Tracking This task aimed to measure precision of motor control without continuous adaptation required (Rommelse et al. 2007c). The stimulus consisted of an inner and outer circle (radius 7.5 and $8.5 \mathrm{~cm}$, respectively). Children were instructed to trace an invisible midline (radius $8 \mathrm{~cm}$ ) between the inner and outer circle as quickly and precisely as possible with a mouse cursor. One practice and one experimental session were administered for both hands separately (clockwise with the right hand and counter clockwise with the left hand). Administration took about $3 \mathrm{~min}$. The dependent measure was the precision (mean distance to midline in $\mathrm{mm}$ averaged across 60 equal parts of the circle) of the left hand. Previous results have shown that precision of the left hand showed endophenotypic-like characteristics (Rommelse et al. 2007c).

Tapping This task measured variability of self-generated motor output (Rommelse et al. 2008a). Children were required to tap as frequently as possible within a certain time period. During tapping, the number of taps was continuously counted and displayed on the screen. One practice session $(5 \mathrm{~s})$ and one experimental session (18 $\mathrm{s})$ 
were administered for both hands separately. The task was first practised and executed with the index finger of the non-preferred hand, thereafter practised and executed with the index finger of the preferred hand. Administration took about $3 \mathrm{~min}$. The dependent measure was the variability (SD of intertap intervals in ms) averaged across hands. Previous results have shown that this measure correlates between siblings (Rommelse et al. 2008a).

Baseline Speed This task was designed to measure variability on a simple reaction time task (Rommelse et al. 2008a). Stimuli consisted of a fixation cross in the centre of a computer screen that changed unpredictably into a white square. Immediately following the response, the white square changed back into the fixation cross. The time interval between a response and the emergence of the next white square varied randomly between 500 to $2,500 \mathrm{~ms}$ in order to prevent anticipation strategies. Subjects were required to press a key as quickly as possible when the white square appeared. A practice session (10 trials) and an experimental session (32 trials) were administered for both hands separately. The task was first practised and executed with the index finger of the non-preferred hand, thereafter practised and executed with the index finger of the preferred hand. Administration took about $5 \mathrm{~min}$. Dependent measure was the variability (SD of reaction times in $\mathrm{ms}$ ) of responses averaged across hands. Previous results have shown that this measure was associated with ADHD and correlates between siblings (Rommelse et al. 2008a).

Motor Timing This task was designed to measure variability of motor timing (Rommelse et al. 2008a). In this task a $1 \mathrm{~s}$ interval had to be produced. The start of the interval was announced by a tone $(80 \mathrm{db}, 50 \mathrm{~ms})$. After the subject's response, visual feedback was given, indicating whether the response was correct, too short or too long. A response was regarded as correct, if it fell between the lower and upper boundary set by a dynamic tracking algorithm. Boundaries were set at 500 to $1,500 \mathrm{~ms}$ at the beginning of the task. If the response fell within these boundaries, the boundaries for the subsequent trial were narrowed by $100 \mathrm{~ms}$. Likewise, the boundaries of the subsequent trial were widened with $100 \mathrm{~ms}$, if the response on the previous trial fell outside those boundaries. Subjects were instructed to produce as accurately as possible the $1 \mathrm{~s}$ interval. Twenty practice trials and 80 experimental trials were administered. Both sessions were preceded by presenting 10 times a cartoon figure for exactly $1 \mathrm{~s}$ on the screen to demonstrate the duration of $1 \mathrm{~s}$. Administration took about $8 \mathrm{~min}$. The dependent measure was the variability (SD of productions in $\mathrm{ms}$ ). Previous results have shown this measure to be a viable endophenotypic candidate (Rommelse et al. 2008a).
Procedure

Administration of the neuropsychological tasks in children with ADHD and their siblings took place at the VU University Amsterdam or at the Radboud University Nijmegen Medical Centre and was conducted simultaneously for all children in a family. Psychostimulants were discontinued for at least $48 \mathrm{~h}$ before testing took place (Pelham et al. 1999). Children were motivated with small breaks. At the end of the session, a gift worth approximately $€ 4$,- -was given. Control children were tested in a similar way in a quiet room at their school. The study had medical-ethical approval.

\section{Data Analyses}

Measures with less than 5\% missing data were subjected to expectation maximization to replace the missing data (Tabachnick and Fidell 2001). Percentages of missing data of the Stop Task (9.2\%) and GMO (8.3\%) were slightly too large to replace. All measures were subjected to a Van der Waerden transformation (Lehmann 1975) to normalize the measures and to depict all measure on the same scale ( $z$-scores) (see Fig. 1). Some measures were mirrored, so that the scores of all variables would imply the same: Higher scores are indicative of poor performance or reflect more severe comorbid problems.

All analyses were carried out on combined parent and teacher ratings. However, since rater-specific results may also be of interest, these results are presented as supplementary material. Any noteworthy differences are described. In order to obtain a reduced number of neuropsychological variables and a more robust neuropsychological construct, two principal component analyses were performed: one on the four EF measures and one on the five motor measures. All four EF measures were related to one major component, explaining 59\% of the task variance (EF-endophenotype explained the following percentages of variance in the individual task measures: $55 \%$ of Stop task; $59 \%$ of Digit Span; $65 \%$ of Visual-spatial sequencing; $57 \%$ of Shifting attentional set). All five motor measures were related to one major component explaining $52 \%$ of the variance (motor-endophenotype explained the following percentages of variance in the individual task measures: $71 \%$ of Pursuit; $46 \%$ of Tracking; $41 \%$ of Tapping; $48 \%$ of Baseline speed; $54 \%$ of Motor timing). In both analyses, additional components did not have an eigenvalue greater than 1 . Therefore, the following results report only on both the main factorial components, which are labelled the 'EFendophenotype' and the 'motor-endophenotype'.

Analyses were performed in SPSS version 15 and in SAGE (Statistical Analysis for Genetic Epidemiology version 5.4, 2007). The latter corrects for relatedness of measurements since more than one child per family participated. The following two terms were used: correlation (referring to a correlation between two variables in the 
Fig. 1 Normalized and standardized measures of ADHD, comorbid problems, and the EF- and motor endophenotypes (aggregated scores of respectively four and five neuropsychological task variables)
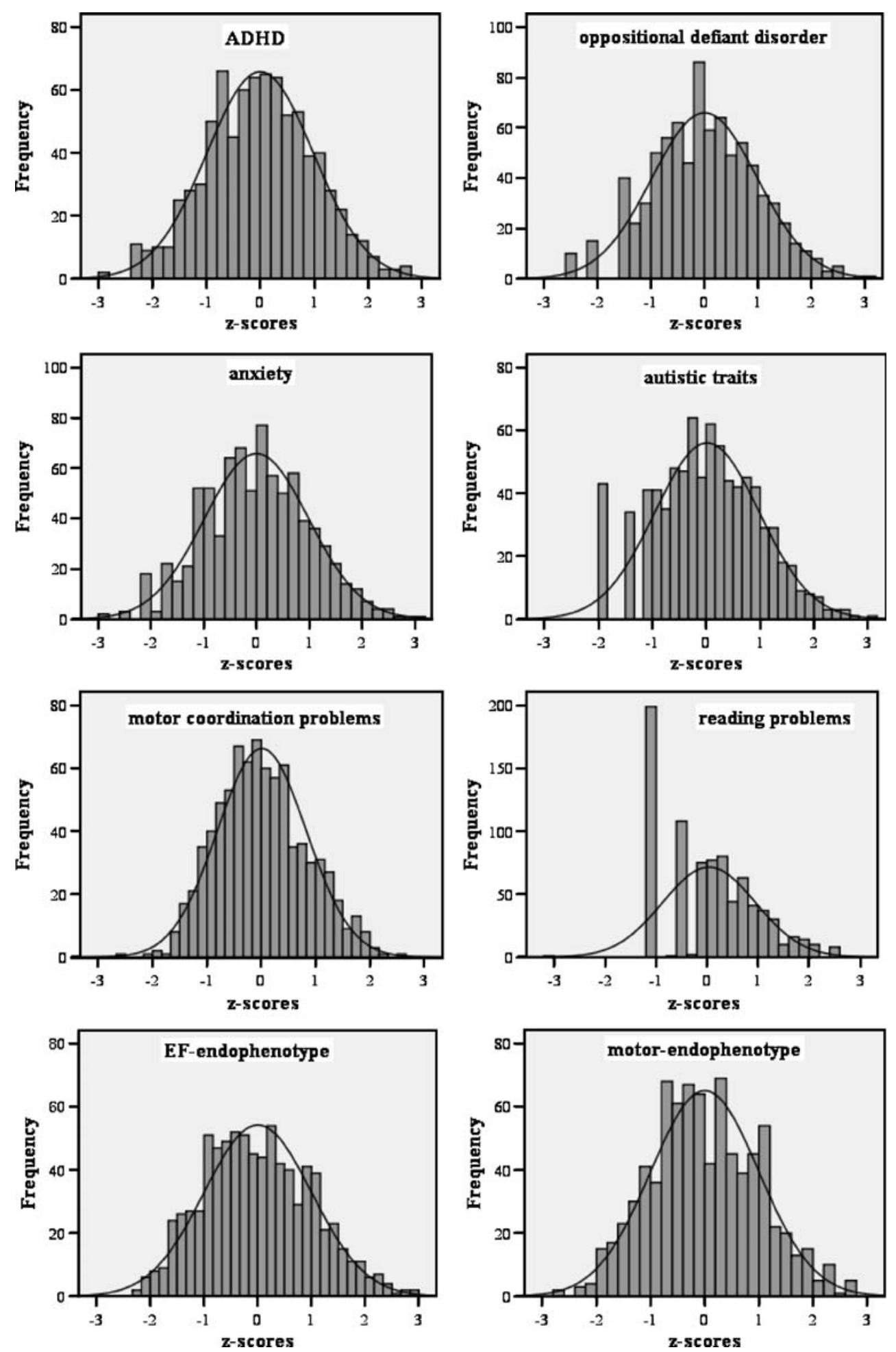

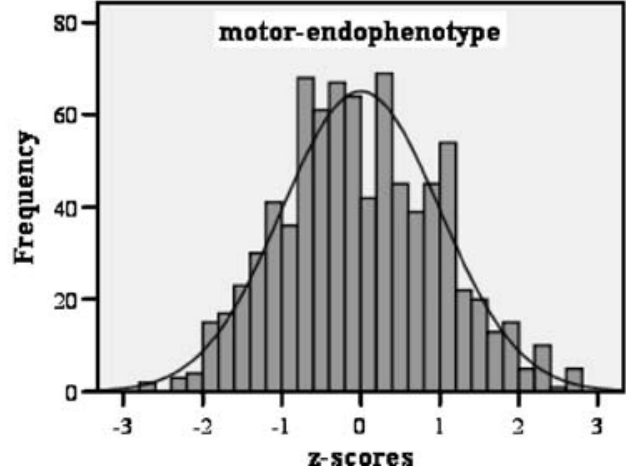

same subject), and sibling cross-correlation (referring to a correlation between siblings for two different variables). In all analyses, IQ, age and sex were used as covariates. Correction for multiple comparisons was applied using the False Discovery Rate (FDR) controlling procedure with a $q$-value of 0.05 (Benjamini and Hochberg 1995). Correlations were interpreted according to Cohen's guidelines (Cohen
1988): 0.10-0.29 (small/modest), 0.30-0.49 (medium/ moderate), and 0.50-1.0 (large/strong).

Concerning the first research aim, correlations were calculated between ADHD and the comorbid problems. Correlation coefficients were compared using dependent correlation twosided $t$-tests (Chen and Popovich 2002). Concerning the second research aim, correlations and sibling cross- 
correlations were calculated between the EF-and motorendophenotypes on the one hand and the measures of comorbid problems on the other hand, in order to examine whether the endophenotypes were related to comorbid problems. The partial correlations and sibling crosscorrelations were calculated, allowing investigation of whether or not the association between the EF-and motorendophenotypes and the comorbid problems was present independent of ADHD. Partial correlations between the endophenotypes and comorbid problems were calculated by adjusting for the correlations endophenotype-ADHD and ADHD-comorbid problem. Partial sibling cross-correlations between endophenotypes and comorbid problems were calculated by correcting for the sibling cross-correlations endophenotype-ADHD and ADHD-comorbid problem. Regarding the third research aim, it was examined whether the comorbid condition could be considered a third, independent condition (Caron and Rutter 1991). If so, the interaction term would have to contribute to prediction of the endophenotype beyond the independent effects of ADHD and the comorbid condition (Baron and Kenny 1986). The interaction score was obtained by a multiplication of the z-score of the ADHD measure with the z-score of a comorbid measure.

\section{Results}

Degree of Association between ADHD and Comorbid Problems

Correlations indicated a strong association between ADHD and oppositional defiant behaviors $(r=0.71, p<0.001)$ and autistic traits $(r=0.69, p<0.001)$. A medium association was found with motor coordination problems $(r=0.53$, $p<0.001)$, anxiety $(r=0.44, p<0.001)$, and reading problems $(r=0.38, p<0.001)$. The correlation between ADHD and oppositional behaviors was nominally significantly stronger than the correlation between ADHD and autistic traits $(t=2.21, p=0.03)$, and significantly stronger than the correlation between ADHD and motor coordination problems, anxiety, and reading problems $(t=8.48,10.73$, and 11.98 , respectively, $p<0.001)$. The correlation between ADHD and autistic traits was stronger than the correlations of ADHD with motor coordination problems, anxiety, and reading problems $(t=7.28,8.88$, and 10.85 , respectively, $p<0.001)$. Motor coordination problems were significantly more correlated with ADHD compared to reading problems ( $t=4.04, p=0.001)$, but not compared to anxiety $(t=1.91$, $p=0.06$ ). The difference between the correlations of anxiety and reading problems with ADHD did not survive correction for multiple testing ( $t=1.98, p=0.05)$. Rater-specific results are presented in supplementary Table 1 .

\section{Relation between the EF-and Motor Endophenotypes and Comorbid Problems}

Almost similar results were obtained for the EF-and motor-endophenotype: both were modestly to moderately correlated with autistic traits, motor coordination problems and reading problems, also after adjustment for ADHD. However, both endophenotypes were not (or only very weakly) related to oppositional defiant behaviours and anxiety, the more so after adjustment for ADHD (see Table 1). Both endophenotypes cross-correlated modestly

Table 1 Correlations and Sibling Cross-correlations between the EF-and Motor Endophenotypes and Comorbid Problems

\begin{tabular}{|c|c|c|c|c|}
\hline & \multicolumn{2}{|c|}{ Correlations $^{\mathrm{a}}$} & \multicolumn{2}{|c|}{ Sibling cross-correlations ${ }^{b}$} \\
\hline & $\begin{array}{l}\mathrm{EF} \\
r \backslash r_{p}^{\mathrm{c}}\end{array}$ & $\begin{array}{l}\text { Motor } \\
r \backslash r_{p}^{\mathrm{c}}\end{array}$ & $\begin{array}{l}\mathrm{EF} \\
r \backslash r_{p}^{\mathrm{d}}\end{array}$ & $\begin{array}{l}\text { Motor } \\
r \backslash r_{p}^{\mathrm{d}}\end{array}$ \\
\hline Oppositional defiant behavior & $\mathbf{0 . 2 3} \backslash 0.00$ & $\mathbf{0 . 2 2} \backslash 0.02$ & $0.09 \backslash 0.03$ & $0.07 \backslash 0.05$ \\
\hline Anxiety & $\mathbf{0 . 1 8} \backslash 0.05$ & $\mathbf{0 . 1 7} \backslash 0.05$ & $0.08 \backslash 0.04$ & $0.06 \backslash 0.05$ \\
\hline Autistic traits & $0.30 \backslash 0.13$ & $0.29 \backslash 0.14$ & $0.27 \backslash 0.20$ & $0.24 \backslash 0.22$ \\
\hline Motor coordination problems & $0.25 \backslash 0.20$ & $0.31 \backslash 0.20$ & $0.19 \backslash 0.15$ & $0.17 \backslash 0.15$ \\
\hline Reading problems & $0.35 \backslash 0.26$ & $0.26 \backslash 0.17$ & $0.29 \backslash 0.19$ & $0.23 \backslash 0.17$ \\
\hline
\end{tabular}

All correlations corrected for age, IQ and sex

Bold correlations were significant after correction for multiple testing.

${ }^{a}$ Correlation endophenotype-comorbid problem measured in the same subject based on 816 children (affected children, non-affected siblings, and controls) corrected for the non-independency of children

b Correlation endophenotype-comorbid problem measured in two different subjects (siblings) based on 540 sibling-pairs (including affected children, non-affected siblings, and controls) corrected for the non-independency of sibling pairs

c $r_{p}=$ Partial correlation endophenotype-comorbid problem corrected for the correlations endophenotype-ADHD and ADHD-comorbid problem

d $r_{p}=$ Partial sibling cross-correlation endophenotype-comorbid problem corrected for the sibling cross-correlations endophenotype-ADHD and ADHD-comorbid problem 
to autistic traits, motor coordination problems and reading problems in siblings, also after adjustment for ADHD. No such findings were found for oppositional defiant behaviours and anxiety, suggesting these comorbid problems did not arise from similar EF and motor deficits as ADHD. Raterspecific results are presented in supplementary Table 2. The only noteworthy difference was that the correlation between the EF-endophenotype and motor coordination problems as reported by teachers became non-significant after correction for ADHD.

EF - and Motor-Endophenotypes in Relation to ADHD with Comorbid Problems

It was examined whether the comorbid condition could be considered a third, independent condition by examining the additional predictive value of the interaction term on the EF and motor components beyond the independent effects of ADHD and the comorbid condition in a linear regression analysis. In none of the regression analyses, did the interaction term explain additional variance of the $\mathrm{EF}$ - or motor-endophenotype beyond the effects of ADHD and the comorbid condition (with $\Delta R^{2}$ values ranging from 0.001 to 0.003 and $p$-values ranging from 0.12 to 0.95 ). Raterspecific results were similar.

\section{Discussion}

The aim of this study was threefold. First, to examine the degree of association between ADHD and comorbid problems (oppositional defiant behaviors, anxiety, autistic traits, motor coordination problems, and reading problems) in order to determine which comorbid problems were most important in relation to ADHD. Second, to examine whether an EF and a motor functioning endophenotype were related to comorbid problems (with and without adjusting for their interdependence on ADHD). Third, to determine whether $\mathrm{EF}$ - and motor-endophenotypes supported the hypothesis that ADHD with comorbid problems is a qualitatively different phenotype than ADHD without comorbid problems.

With respect to the first aim of our study, the various comorbid problems were all significantly associated with ADHD. That is, having a more severe form of ADHD was related to having more severe oppositional defiant behaviors, higher levels of anxiety, more autistic traits, and more severe motor coordination and reading problems, which concurs with previous studies reporting on various disorders in combination with ADHD (Angold et al. 1999; Bauermeister et al. 2007; Gillberg et al. 2004). Oppositional defiant behaviors and autistic traits were most strongly correlated with ADHD severity compared to the other investigated comorbid problems. The former finding is well established in literature (Angold et al. 1999; Bauermeister et al. 2007; Gillberg et al. 2004; Jensen et al. 1997). Since oppositional defiant behaviors with ADHD may significantly influence the type of treatment that can be best prescribed (Hechtman et al. 2005; Jensen et al. 2001), it is important to assess oppositional defiant behavior in ADHD-patients. The latter finding (ADHD in combination with autistic traits) is still controversial, since the $D S M-I V$ does not support a diagnostic combination of ADHD and autistic disorder (Corbett and Constantine 2006; Goldstein and Schwebach 2004). This may need to be reconsidered when developing the future $D S M$, since our findings and numerous previous findings suggest that ADHD is frequently associated with characteristics of autistic disorder (Corbett and Constantine 2006; Goldstein and Schwebach 2004; Leyfer et al. 2006). Furthermore, ADHD correlated moderately with motor coordination problems and reading problems. Since both motor coordination problems and reading problems can significantly interfere with academic functioning in a child that is already vulnerable to academic failure because of ADHD symptoms (Rasmussen and Gillberg 2000), it is important to determine vulnerabilities of motor coordination and reading in children in ADHD assessment. Anxiety was moderately correlated with ADHD, to a comparable degree as motor coordination problems and reading problems. Anxiety as internalizing disorder may be an easily overlooked comorbid problem in children with ADHD given the externalizing nature of ADHD symptoms. However, since comorbid anxiety may influence response to methylphenidate (Pliszka 1989; Tannock et al. 1995) and is predictive of a range of psychiatric disorders in adolescence (Bittner et al. 2007), assessment of anxiety in children with ADHD is important (Angold et al. 1999; Bauermeister et al. 2007; Gillberg et al. 2004).

Second, comorbid problems were not merely associated phenotypically, but autistic traits, motor coordination problems and reading problems were also related to the EF and motor ADHD-endophenotypes after correction for ADHD. These findings may pinpoint to a shared underlying general neuropsychological dysfunctions that may give rise to both ADHD and several associated domains. Further support for this hypothesis was found, when the sibling cross-correlations between the endophenotypes and the comorbid problems were calculated and were found to be significant for these problem domains. This suggests that these comorbid problems may arise from similar EF and motor deficits that are related to ADHD. It thus appears that autistic traits, motor coordination problems and reading problems share familial factors that give rise to EF and motor functioning problems also apparent in ADHD (Pennington 2006). These familial neuropsychological endophenotypes appear to have multiple behavioral consequences (pleiotrophy) (Banaschewski et al. 
2005). The chain of events leading to ADHD symptoms does not appear to be independent of the sequence of events leading to these other domains. Atypical brain development may be the basis of developmental disorders, resulting in frequent co-occurrence of multiple disorders in the same child (Kaplan et al. 2001; Pennington, 2006). However, no such evidence was found for oppositional defiant behaviors and anxiety in relation to ADHD. Their relation with the endophenotypes disappeared after correction for ADHD and no sibling cross-correlations were found between these problem domains and the endophenotypes. This suggests that the occurrence of these problems in children with ADHD may be merely phenotypical. That is, the occurrence of oppositional defiant behaviors and anxiety in patient with ADHD may be secondarily caused by having ADHD and not related to similar familial EF and motor underpinnings.

Results for the third aim of our study revealed that the specific combination of ADHD with a comorbid problem may not be best seen as forming a distinct phenotype (Banaschewski et al. 2005; Biederman et al. 1991; Caron and Rutter 1991; Jensen et al. 2001), since the interaction term between ADHD and the comorbid condition did not have predictive value on the EF and motor components beyond the independent effects of ADHD and the comorbid condition (Baron and Kenny 1986). This suggested that larger endophenotypic dysfunctions resulted in 'more of the same' (a more severe form of ADHD and comorbid problem) but not in a phenotypically distinct subtype (Banaschewski et al. 2005; Biederman et al. 1991; Caron and Rutter 1991; Jensen et al. 2001). Based on these preliminary results obtained using questionnaires, it does not appear necessary to define new diagnostic categories in a future $D S M$ entailing ADHD with specific comorbid problems. However, given the limitations of our study described below and evidence from other research groups suggesting that ADHD with comorbid conditions is different from "pure" ADHD, further research is warranted.

Several limitations of this study warrant consideration. First of all, questionnaires were used to gather information on comorbid problems. Although questionnaires may more aptly reflect the underlying continuously distributed nature of disorders, they do not provide sufficient information to diagnose a child according to $D S M-I V$ criteria. Additional measures that might have allowed us to diagnose all children for comorbid problems would have added to the study findings. In addition, using questionnaires may bias the results towards showing higher degrees of comorbidity. However, this does not explain the relative differences in phenotypic association between ADHD and the various comorbid problems. A second limitation that should be noted, is that our sample was biased towards having ADHD (or being biologically related to someone who does) and biased towards not having ADHD. Although the whole range of ADHD severity and severity of comorbid problems was represented in our sample, the current findings on the associations between the comorbid problems may, therefore, not be applicable to the occurrence of these symptoms in the general population. Third, sibling correlations were only modest. This suggests that the familial effects on the neuropsychological measures are modest. However, we did not expect to find large correlations. It is likely that multiple genes relate to the ADHD phenotype (polygenetically determined disorder), each having a small effect (Faraone and Biederman, 1998) with no single gene being necessary or sufficient to cause ADHD. It was expected that sibling correlations for familially determined neuropsychological deficits would also be small, let alone sibling cross-correlations (sibling correlations for different measures). In addition, previous research on neuropsychological functioning in patients with ADHD has shown that a substantial proportion of patients does not perform abnormally on neuropsychological measures (Nigg et al. 2005), resulting in an overall small association between neuropsychological deficits and ADHD. Nevertheless, we found consistent correlational patterns: the EF and motor endophenotype cross-correlated significantly (after stringent correction for multiple testing) in siblings to autistic traits, motor coordination problems and reading problems. Neither of the endophenotypes showed this pattern of results for oppositional defiant behaviors and anxiety, suggesting the occurrence of oppositional defiant behaviors and anxiety in patient with ADHD may be secondarily caused by having ADHD and not related to similar familial, EF and motor underpinnings. A fourth limitation may be related to the neuropsychological battery used in this study, which tapped into executive and motor functions, but not into other important domains in relation to ADHD, such as visuoconstructive or language related functions. Replicating this study using other neuropsychological constructs, will offer the possibility to examine the generalization of the current findings to other neuropsychological domains. A fifth limitation is related to the fact that children with a full autistic disorder were excluded from the study, since these children cannot be diagnosed with ADHD according to the $D S M-I V$. Our findings on autistic traits are thus limited to children with pervasive developmental disorder not otherwise subscribed (PDD-NOS).

Understanding the presence of comorbidity between psychiatric conditions offers a mean of correcting and validating psychiatric nosology (Angold et al. 1999). The co-occurrence of ADHD and comorbid problems is evident and arises partly from shared familial/heritable neuropsychological EF and motor dysfunctions, suggesting these symptoms cannot be viewed, diagnosed or treated independently of one another. This may have implications for the theoretical background on which the development of a 
future $D S M$ as well as for the diagnostic and treatment procedures utilized in daily clinical practice. With respect to the latter, these findings suggest always assessing possible comorbid conditions in addition to ADHD, even when symptoms of ADHD appear the most prominent. It appears, however, that based on the preliminary findings of the current study, new diagnostic categories in a future $D S M$ entailing ADHD with specific comorbid problems are not necessary.

Acknowledgements The authors thank all of the parents, teachers, and children who participated. This study was partly funded by a grant assigned to Stephen Faraone by the National Institute of Mental Health (NIH grant \# R01 MH62873-01A1). The results of this paper were obtained by using the program package S.A.G.E., which is supported by a U.S. Public Health Service Resource Grant (RR03655) from the National Center for Research Resources.

Open Access This article is distributed under the terms of the Creative Commons Attribution Noncommercial License which permits any noncommercial use, distribution, and reproduction in any medium, provided the original author(s) and source are credited.

\section{References}

American Psychiatric Association. (1994). Diagnostic and Statistical Manual for Mental Disorders (4th ed.). Washington, DC: American Psychiatric.

Angold, A., Costello, E. J., \& Erkanli, A. (1999). Comorbidity. Journal of Child Psychology and Psychiatry, and Allied Disciplines, 40, 57-87. doi:10.1111/1469-7610.00424.

Banaschewski, T., Hollis, C., Oosterlaan, J., Roeyers, H., Rubia, K., Willcutt, E., et al. (2005). Towards an understanding of unique and shared pathways in the psychopathophysiology of ADHD. Developmental Science, 8, 132-140. doi:10.1111/j.1467-7687.2005.00400.x.

Baron, R. M., \& Kenny, D. A. (1986). The moderator-mediator variable distinction in social psychological research: conceptual, strategic, and statistical considerations. Journal of Personality and Social Psychology, 51, 1173-1182. doi:10.1037/0022-3514.51.6.1173.

Bauermeister, J. J., Shrout, P. E., Ramírez, R., Bravo, M., Alegría, M., Martínez-Taboas, A., et al. (2007). ADHD correlates, comorbidity, and impairment in community and treated samples of children and adolescents. Journal of Abnormal Child Psychology, 35, 883-898. doi:10.1007/s10802-007-9141-4.

Benjamini, Y., \& Hochberg, Y. (1995). Controlling the false discovery rate: a practical and powerful approach to multiple testing. Journal of the Royal Statistical Society: Series B, 57, 289-300.

Berument, S. K., Rutter, M., Lord, C., Pickles, A., \& Bailey, A. (1999). Autism screening questionnaire: diagnostic validity. The British Journal of Psychiatry, 175, 444-451. doi:10.1192/bjp.175.5.444.

Bhatia, M., Nigam, V., Bohra, N., \& Malik, S. (1991). Attention deficit disorder with hyperactivity among paedriatic outpatients. Journal of Child Psychology and Psychiatry, and Allied Disciplines, 32, 297-306. doi:10.1111/j.1469-7610.1991.tb00308.x.

Biederman, J. (2004). Impact of comorbidity in adults with attentiondeficit/hyperactivity disorder. The Journal of Clinical Psychiatry, 65(Supplement 3), 3-7.

Biederman, J., Newcorn, J., \& Sprich, S. (1991). Comorbidity of attention deficit hyperactivity disorder with conduct, depressive, anxiety, and other disorders. The American Journal of Psychiatry, 148, 564-577.

Biederman, J., Faraone, S. V., Milberger, S., Curtis, S., Chen, L., Marrs, A., et al. (1996). Predictors of persistence and remission of ADHD: results from a four-year prospective follow-up study of ADHD children. Journal of the American Academy of Child and Adolescent Psychiatry, 35, 343-351.

Biederman, J., Monuteaux, M. C., Kendrick, E., Klein, K. L., \& Faraone, S. V. (2005). The CBCL as a screen for psychiatric comorbidity in paediatric patients with ADHD. Archives of Disease in Childhood, 90, 1010-1015. doi:10.1136/adc.2004.056937.

Bittner, A., Egger, H. L., Erkanli, A., Costello, E. J., Foley, D. L., \& Angold, A. (2007). What do childhood anxiety disorders predict? Journal of Child Psychology and Psychiatry, and Allied Disciplines, 48, 1174-1183.

Brookes, K., Xu, X., Chen, W., Zhou, K., Neale, B., Lowe, N., et al. (2006). The analysis of 51 genes in DSM-IV combined type attention deficit hyperactivity disorder: association signals in DRD4, DAT1 and 16 other genes. Molecular Psychiatry, 11, 934-953. doi:10.1038/sj.mp.4001869.

Caron, C., \& Rutter, M. (1991). Comorbidity in child psychopathology: concepts, issues and research strategies. Journal of Child Psychology and Psychiatry, and Allied Disciplines, 32, 1063-1080. doi:10.1111/j.1469-7610.1991.tb00350.x.

Chen, P. Y., \& Popovich, P. M. (2002). Correlation: Parametric and nonparametric measures. Thousand Oaks, CA: Sage Publications.

Clark, T., Feehan, C., Tinline, C., \& Vostanis, P. (1999). Autistic symptoms in children with attention deficit hyperactivity disorder. European Journal of Child and Adolescent Psychiatry, 8, 50-55. doi:10.1007/s007870050083.

Cohen, J. (1988). Statistical power analysis for the behavioral sciences (2nd ed.). Hillsdale, NJ: Erlbaum.

Conners, K. (1996). Rating scales in ADHD. Durham, North Carolina: Duke University Medical Center.

Connor, D. F., Edwards, G., Fletcher, K. E., Baird, J., Barkley, R. A., \& Steingard, R. J. (2003). Correlates of comorbid psychopathology in children with ADHD. Journal of the American Academy of Child and Adolescent Psychiatry, 42, 193-200. doi:10.1097/ 00004583-200302000-00013.

Corbett, B. A., \& Constantine, L. J. (2006). Autism and attention deficit hyperactivity disorder: assessing attention and response control with the integrated visual and auditory continuous performance test. Child Neuropsychology, 12, 335-348. doi:10.1080/09297040500350938.

Cunningham, C. E., \& Boyle, M. H. (2002). Preschoolers at risk for attention-deficit hyperactivity disorder and oppositional deviant disorder: family, parenting, and behavioral correlates. Journal of Abnormal Child Psychology, 30, 555-569. doi:10.1023/ A: 1020855429085 .

Faraone, S. V., \& Biederman, J. (1998). Neurobiology of attentiondeficit hyperactivity disorder. Biological Psychiatry, 44, 951958. doi:10.1016/S0006-3223(98)00240-6.

Gillberg, C., Gillberg, I. C., Rasmussen, P., Kadesjö, B., Söderström, H., Råstam, M., et al. (2004). Co-existing disorders in ADHDimplications for diagnosis and intervention. European Journal of Child and Adolescent Psychiatry, 13, 80-92. doi:10.1007/ s00787-004-1008-4.

Goldstein, S., \& Schwebach, A. J. (2004). The comorbidity of pervasive developmental disorder and attention deficit hyperactivity disorder: results of a retrospective chart review. Journal of Autism and Developmental Disorders, 34, 329-339. doi:10.1023/ B:JADD.0000029554.46570.68.

Goodman, R. (1997). The strengths and difficulties questionnaire: a research note. Journal of Child Psychology and Psychiatry, and Allied Disciplines, 38, 581-586. doi:10.1111/j.1469-7610.1997.tb01545.x.

Hartman, C. A., Luteijn, E., Serra, M., \& Minderaa, R. (2006). Further investigation of the construct validity of the children's social behavior questionnaire (CSBQ): an instrument for children with milder forms of PDD. Journal of Autism and Developmental Disorders, 36, 325-342. doi:10.1007/s10803-005-0072-z. 
Hechtman, L., Etcovitch, J., Platt, R., Arnold, L., Abikoff, H., Newcorn, J., et al. (2005). Does multimodal treatment of ADHD decrease other diagnoses? Clinical Neuroscience Research, 5, 273-282. doi:10.1016/j.cnr.2005.09.007.

Jensen, P. S., Martin, D., \& Cantwell, D. P. (1997). Comorbidity in ADHD: implications for research, practice, and DSM-V. Journal of the American Academy of Child and Adolescent Psychiatry, 36, 1065-1079.

Jensen, P. S., Hinshaw, S. P., Kraemer, H. C., Lenora, N., Newcorn, J. H., Abikoff, H. B., et al. (2001). ADHD comorbidity findings from the MTA study: comparing comorbid subgroups. Journal of the American Academy of Child and Adolescent Psychiatry, 40, 147-158. doi:10.1097/00004583-200102000-00009.

Kalverboer, A. F., \& Van Dellen, C. (1990). Groningen Motor Observation Scale (GMOS). In A. F. Kalverboer (Ed.), Developmental biopsychology: Experimental and observational studies in children at risk. Ann Arbor: The University of Michigan Press.

Kaplan, B. J., Dewey, D. M., Crawford, S. G., \& Wilson, B. N. (2001). The term comorbidity is of questionable value in reference to developmental disorders: data and theory. Journal of Learning Disabilities, 34, 555-565. doi:10.1177/002221940103400608.

Kessler, R. C., Adler, L., Barkley, R., Biederman, J., Conners, C. K., Demler, O., et al. (2006). The prevalence and correlates of adult ADHD in the United States: results from the national comorbidity survey replication. The American Journal of Psychiatry, 163, 716723. doi:10.1176/appi.ajp.163.4.716.

Lehmann, E. L. (1975). Nonparametrics: Statistical methods based on ranks. San-Francisco: Holden-Day.

Leyfer, O. T., Folstein, S. E., Bacalman, S., Davis, N. O., Dinh, E., Morgan, J., et al. (2006). Comorbid psychiatric disorders in children with autism: interview development and rates of disorders. Journal of Autism and Developmental Disorders, 36, 849-861. doi:10.1007/s10803-006-0123-0.

Nigg, J. T., Willcutt, E. G., Doyle, A. E., \& Sonuga-Barke, E. J. (2005). Causal heterogeneity in attention-deficit/hyperactivity disorder: do we need neuropsychologically impaired subtypes? Biological Psychiatry, 57, 1224-1230. doi:10.1016/j.biopsych.2004.08.025.

Owens, J. (2005). The ADHD and sleep conundrum: a review. Journal of Developmental and Behavioral Pediatrics, 26, 312322. doi:10.1097/00004703-200508000-00011.

Pelham, W. E., Aronoff, H. R., Midlam, J. K., Shapiro, C. J., Gnagy, E. M., Chronis, A. M., et al. (1999). A comparison of Ritalin and Adderall: efficacy and time-course in children with attentiondeficit/hyperactivity disorder. Pediatrics, 103, e43. doi:10.1542/ peds.103.4.e43.

Pennington, B. F. (2006). From single to multiple deficit models of developmental disorders. Cognition, 101, 385-413. doi:10.1016/ j.cognition.2006.04.008.

Pliszka, S. R. (1989). Effect of anxiety on cognition, behavior, and stimulant response in ADHD. Journal of the American Academy of Child and Adolescent Psychiatry, 28, 882-887. doi:10.1097/ 00004583-198911000-00012.

Rasmussen, P., \& Gillberg, C. (2000). Natural outcome of ADHD with developmental coordination disorder at age 22 years: a controlled, longitudinal, community-based study. Journal of the American Academy of Child and Adolescent Psychiatry, 39, 1424-1431. doi:10.1097/00004583-200011000-00017.
Reich, T., Cloninger, C., \& Guze, S. (1975). The multifactoral model of disease transmission: I. Description of the model and its use in psychiatry. The British Journal of Psychiatry, 127, 1-10. doi:10.1192/bjp. 127.1.1.

Rommelse, N. N., Altink, M. E., De Sonneville, L. M., Buschgens, C. J., Buitelaar, J., Oosterlaan, J., et al. (2007a). Are motor inhibition and cognitive flexibility dead ends in ADHD? Journal of Abnormal Child Psychology, 35, 957-967. doi:10.1007/ s10802-007-9146-z.

Rommelse, N. N., Oosterlaan, J., Buitelaar, J., Faraone, S. V., \& Sergeant, J. A. (2007b). Time reproduction in children with ADHD and their non-affected siblings. Journal of the American Academy of Child and Adolescent Psychiatry, 46, 582-590. doi:10.1097/CHI.0b013e3180335af7.

Rommelse, N. N., Altink, M. E., Oosterlaan, J., Buschgens, C. J., Buitelaar, J., De Sonneville, L. M., et al. (2007c). Motor control in children with ADHD and non-affected siblings: deficits most pronounced using left hand. Journal of Child Psychology and Psychiatry, and Allied Disciplines, 48, 1071-1079. doi:10.1111/ j.1469-7610.2007.01781.x.

Rommelse, N. N., Altink, M. E., Oosterlaan, J., Beem, L., Buschgens, C. J., Buitelaar, J., et al. (2008a). Speed, variability, and timing of motor output in ADHD: which measures are useful for endophenotypic research? Behavior Genetics, 38, 121-132. doi:10.1007/s10519-007-9186-8.

Rommelse, N. N., Altink, M. E., Oosterlaan, J., Buschgens, C. J., Buitelaar, J., \& Sergeant, J. A. (2008b). Support for an independent familial segregation of executive and intelligence endophenotypes in ADHD-families. Psychological Medicine, 38 , 1595-1606. doi:10.1017/S0033291708002869.

S.A.G.E. 6.0 (2007). Statistical Analysis for Genetic Epidemiology http://darwin.cwru.edu/sage/

Tabachnick, B. G., \& Fidell, L. S. (2001). Using multivariate statistics (4th ed.). Needham Heights: Allyn and Bacon.

Tannock, R., Ickowicz, A., \& Schachar, R. (1995). Differential effects of methylphenidate on working memory in ADHD children with and without comorbid anxiety. Journal of the American Academy of Child and Adolescent Psychiatry, 34, 886-896. doi:10.1097/ 00004583-199507000-00012.

Taylor, E. A. (1986). Childhood hyperactivity. The British Journal of Psychiatry, 149, 562-573. doi:10.1192/bjp.149.5.562.

Waldman, I. D. (2005). Statistical approaches to complex phenotypes: Evaluating neuropsychological endophenotypes for attentiondeficit/hyperactivity disorder. Biological Psychiatry, 57, 13471356. doi:10.1016/j.biopsych.2005.03.002.

Willcutt, E. G., Pennington, B. F., Olson, R. K., Chhabildas, N., \& Hulslander, J. (2005). Neuropsychological analyses of comorbidity between reading disability and attention deficit hyperactivity disorder: in search of the common deficit. Developmental Neuropsychology, 27, 35-78. doi:10.1207/s15326942dn2701_3.

Willcutt, E., Boada, R., Pennington, B., \& Riddle, M. (2009). A parent-report screening questionnaire for learning difficulties in children. In press

Wilson, B. N., Kaplan, B. J., Crawford, S. G., Campbell, A., \& Dewey, D. (2000). Reliability and validity of a parent questionnaire on childhood motor skills. The American Journal of Occupational Therapy., 54, 484-493. 